\title{
A Tunable Fabry-Perot Optical Filter for Application in Biochemical Analysis of Human's Fluids
}

\author{
C. Pinheiro*, J. G. Rocha*, L. M. Goncalves*, S. Lanceros-Mendez** and G. Minas* \\ * University of Minho, Dept. of Industrial Electronics, 4800-058 Guimaraes, Portugal \\ **University of Minho, Dept. of Physics, Campus de Gualtar, 4710-057 Braga, Portugal \\ gminas@dei.uminho.pt
}

\begin{abstract}
This paper reports a Fabry-Perot tunable optical filter for application in clinical analysis, especially to measure the concentration of biochemical substances in biological fluids. Its tunable characteristics enable the measurement of different biochemical substances with the same device. It is composed of two parallel thin silver mirrors with a $\beta$-PVDF (Poly(Vinylidene Fluoride) in its $\beta$ phase) film in-between as the resonance cavity. The filter is tuned by adjusting the space between the mirrors through an electric voltage inducing dipolar orientation in the $\beta$ PVDF film. Once the thickness changes of the $\beta$-PVDF film is due to variations at a molecular level, the changes are rather homogeneous within the samples. As a result, this approach achieves parallelism between the two mirrors, which is a complex problem to solve in conventional Fabry-Perot optical filters. Moreover, compared with an array of non-tunable optical filters with different cavity lengths, usually used to solve the parallelism problem, this device has a reduced area, its fabrication process is easier and it covers a wider spectral range. In addition, when used as a part of a biological fluids analysis system, it avoids the need for expensive optics and enables low-cost and portable devices, which would improve the use of spectrophotometric analysis in clinical diagnostics.
\end{abstract}

\section{INTRODUCTION}

For diagnostic reasons patients in a hospital are often subjected to biochemical analysis of their biological body fluids, such as blood or urine. Usually the analyses are carried out in clinical laboratories and the results become available after several hours, sometimes days. As a consequence, a reliable diagnosis cannot be performed within the consultation time. Mistakes in the logistics, such as lost samples and mislabeling, may further delay diagnosis [1]. The automated equipment used in a state-of-the-art laboratory reduces errors, but use high sample and reagent volumes, making the analysis systems expensive and does not contribute to patient comfort [2]. Outside the laboratory environment, reagent strips are commercially available [3]. They provide a color when a fluid sample contacts the strip. However, such strips are intended for a limited set of biomolecules to be analyzed and the readout of color is merely qualitative (by visual inspection). The need for rapid and in-situ measurements led to the development of small and portable devices that provide an instantaneous result with the same performance achieved using the state-of-the-art clinical laboratory equipments.

This paper describes a tunable optical filter, based on Fabry-Perot thin films optical resonators, for application in clinical analysis devices, especially for colorimetric measurement, by optical absorption, of the concentration of biochemical substances in biological fluids, featuring white light illumination for the color readout.

\section{BACKGROUND OF COLORIMETRIC ANALYSES}

In clinical diagnostics, the colorimetric detection by optical absorption is the spectrophotometric analysis technique most commonly used to determine the concentration and/or amount of a particular biochemical substance in biological fluids samples [4]. In colorimetric analyses, a fluid sample is mixed with a specific reagent, proper for the biochemical substance being analyzed, and, from that mixture, a colored product is obtained. The intensity of that color is directly proportional to the concentration of the biochemical substance in analysis and the absorption spectra of the mixture show a maximum peak at a specific wavelength [5]. As an example, for determining the total protein concentration in a blood sample the mixture has an absorption maximum at $595 \mathrm{~nm}$ (Fig. 1). The color readout needs a wavelength dependent light source for illumination provided by a monochromator or laser, which are expensive devices. The use of selective optical filters enables measurements using a regular white light source, avoiding the use of those complex and expensive optical systems and ensuring analysis at any location with instantaneous results.

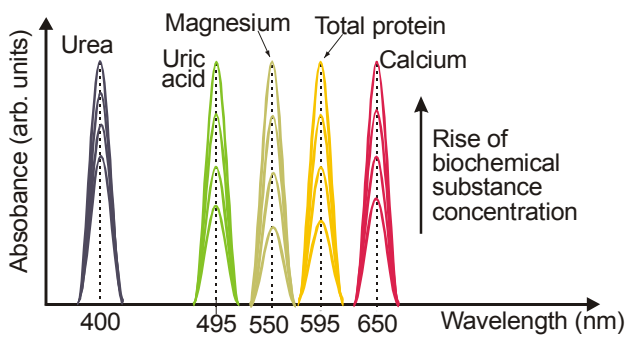

Fig. 1. Absorption spectra shape for some biochemical substances with different concentrations in blood or urine.

The device operation is based on colorimetric detection by optical absorption. A white light impinging spectrum is filtered, by the optical filters, to a narrow spectral band centered at the wavelength at which the colored mixture has its absorption maximum. The intensity of the spectral component transmitted through the colored mixture is measured using a photodiode and readout electronics that allows simpler computer interfacing. The use of tunable optical filters enables 
setting several narrow spectral bands, which allows analyzing several biochemical substances with the same device.

\section{OPTICAL INTERFACES}

This section introduces the basis of the electromagnetic theory and its application in the calculation of the behavior of optical filters based in Fabry-Perot resonant cavities.

\section{A. Principles of the propagation of the electromagnetic waves}

The light is an electromagnetic wave, and the study of its propagation must start with the Maxwell equations. With the objective to become this study not very extensive, it will be started with some results of the Maxwell equations that are valid for linear media. A more detailed and rigorous approach of the electromagnetic field equations can be found in [6].

\section{B. Amplitude of the electromagnetic waves}

An electromagnetic wave can be represented by:

$$
\left\{\begin{array}{l}
\vec{E}=E_{o} e^{j(\omega t-n \cdot K . r)} \\
\vec{H}=H_{o} e^{j(\omega t-n \cdot K \cdot r)}
\end{array}\right.
$$

where $\vec{E}$ and $\vec{H}$ are respectively its electric and magnetic fields, $\omega$ is the optical angular frequency, $K$ is the wave number $(2 \pi / \lambda)$ and $n$ is the refractive index of the propagation medium. If the propagation medium is absorbent, the refractive index must be substituted by $n-j k$, where $k$ is the coefficient of extinction of the medium and $j$ is the complex operator.

For a given wave, the relationship between the electric and magnetic fields is fixed and, in an isotropic medium, is given by:

$$
\vec{H}=\sqrt{\frac{\varepsilon}{\mu}} n \vec{E},
$$

where $\varepsilon$ and $\mu$ are the absolute dielectric and the induction constants of the medium, respectively [7],

$$
\begin{aligned}
& \varepsilon=\varepsilon_{r} \varepsilon_{o}, \\
& \mu=\mu_{r} \mu_{o}, \\
& \varepsilon_{o}=1 /\left(\mu_{o} c^{2}\right), \\
& \mu_{o}=4 \pi \times 10^{-7} \mathrm{NA}^{-2},
\end{aligned}
$$

where $\varepsilon_{r}$ and $\mu_{r}$ are the relative dielectric and the induction constants of the medium, $\varepsilon_{o}$ and $\mu_{o}$ are the dielectric and the induction constants of the free space and $c$ is the speed of the light in vacuum. In an anisotropic media the equation (2) becomes more complex, since $\varepsilon$ and $\mu$ are vectors instead of scalar quantities $[8,9]$.

The directions of the vectors $\vec{E}$ and $\vec{H}$ are also related, forming an angle of $\pi / 2 \mathrm{rad}$ between them and with the direction of propagation. The direction of the propagation is given by the rule of the right hand.

\section{Polarization}

The light that travels in a given direction can have two independent polarizations. The light incident in a surface, forming an angle $\theta$ with its normal, can have either the electric field vector or the magnetic field vector parallel to the plane of incidence. In the first case, the polarization is $p$ and in the second, the polarization is $s$. In a general way, the electric field vector forms an angle $\phi$ with the plane of incidence. In this in case, it can be decomposed in two components, one of polarization $p$ and other of polarization $s$, being:

$$
\begin{array}{ll}
E_{p}=E \cos \phi \cos \theta & \text { for } p \text { polarization } \\
E_{s}=E \sin \phi & \text { for } s \text { polarization. }
\end{array}
$$

In a similar way, for the magnetic field vector:

$$
\begin{aligned}
& H_{p}=H \cos \phi \\
& H_{s}=-H \sin \phi \cos \theta .
\end{aligned}
$$

The normal refractive index $n$ is equal to $H / E$. In a similar way, a generalized refractive index $u$ can be defined for each of the polarizations, such that:

$$
\begin{aligned}
& u_{p}=H_{p} / E_{p}=n / \cos \theta \\
& u_{s}=H_{s} / E_{s}=H \cos \theta / E=-n \cos \theta .
\end{aligned}
$$

Notice that all the polarization states can be decomposed in the $p$ and $s$ components, possibly with a phase shift between them. This turns the following analysis useful for all the polarization states. In a general way, any optic interface has different properties for the two polarizations, except when the incident light is normal to the surface. In this case, the polarizations $p$ and $s$ are equivalent.

\section{Boundary conditions}

The boundary conditions in each interface between two different optical media derive from the electromagnetic theory and allow establishing the relationship between the electromagnetic fields on one side of the interface with the ones on the other side. These conditions state that the components of $E$ and $H$ parallel to the interface must have the same value in both sides.

\section{E. Properties of a thin film stack}

In Fig. 2 a set of thin films with $q$ interfaces, i. e., $q-1$ films is shown.

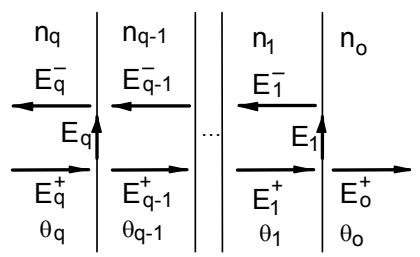

Fig. 2. Thin film stack.

The film $i$ has a wave traveling to the right, $E_{i}^{+}$, and another one traveling to the left, $E_{i}^{-}$. They make an angle $\theta_{i}$ to the normal of the interface. The film has a refractive index $n_{i}$ and a thickness $d_{i}$ (not represented in the figure). Film 0 (at the right side) is the exit medium and the film $q$ (at the left side) is the incidence medium. Usually, the incidence as well as the exit media of a film stack is constituted by air.

Assuming that the stack of thin films forms an optical filter, the beam of incident light is $E_{q}^{+}$, the reflected one is $E_{q}^{-}$and 
the transmitted one is $E_{o}^{+} . E_{o}^{-}$is equal to zero, once it is considered the case in which there is only one light source on the left side of the filter. In the case that several light sources exist, each one can be analyzed separately, once it is estimated that the optical medium is linear and the superposition theorem is valid [10]. From here, only the values of the electric fields $E_{q}^{+}, E_{q}^{-}$and $E_{o}^{+}$are important. All the other fields have interest only for the attainment of the three last ones.

\section{F. Optical path}

The phase thickness of the film $i$ in the case of the light with normal incidence is given by:

$$
g_{i}=\frac{2 \pi u_{i} d_{i}}{\lambda}
$$

where $d_{i}$ is the thickness of the film and $\lambda$ the wavelength of the light in the free space. The phase thickness represents the variation in phase that the light suffers when crosses the film [11]. In the most general case, where the light arrives with an angle $\theta_{i}$, the expression of the phase thickness is:

$$
g_{i}=\frac{2 \pi u_{i} d_{i} \cos \theta_{i}}{\lambda}
$$

where the angles $\theta_{i}$ are determined from the Snell law:

$$
u_{q} \sin \theta_{q}=u_{i} \sin \theta_{i}=u_{o} \sin \theta_{o} .
$$

The variable $u_{i}$ will be $u_{p i}$ in the polarization $p$ and $u_{s i}$ in the polarization $s$. Notice that, in the case of the film $i$ absorbing light, $u_{i}$ is a complex quantity, what makes $i$ a complex number too.

\section{G. Equations in the interface between two films}

First, it is assumed that the phase of the waves is zero in the interface between the films $i$ and $i-1$. In other words, the phase is zero at the right side of the film $i$. In the interface between the film $i+1$ and $i$, at the left side of the interface the fields $E_{i+1}^{+}$and $E_{i+1}^{-}$are present, and at the right side are present the fields $E_{i}^{+} e^{j g_{i}}$ and $E_{i}^{-} e^{-j g_{i}}$, where $e^{j g_{i}}$ and $e^{-j g_{i}}$ represent the phase shifts suffered by the electromagnetic waves along the film $i$.

Using the boundary conditions described previously, it comes to the interface $i+1, i$ :

$$
E_{i+1}^{+}+E_{i+1}^{-}=E_{i}^{+} e^{j g_{i}}+E_{i}^{-} e^{-j g_{i}} .
$$

It is convenient to define now:

$$
E_{i}=E_{i+1}^{+}+E_{i+1}^{-} \text {and } H_{i}=H_{i}^{+}+H_{i}^{-} \text {, }
$$

where $E_{i}$ and $H_{i}$ are the total fields in the interface between the films $i$ and $i-1$. Knowing that $H_{i}^{ \pm}= \pm u_{i} E_{i}^{ \pm}$, then:

$$
\begin{aligned}
& E_{i}^{+}=\frac{1}{2}\left(E_{i}+H_{i} / u_{i}\right) \\
& E_{i}^{-}=\frac{1}{2}\left(E_{i}-H_{i} / u_{i}\right) .
\end{aligned}
$$

Then the equation (3) becomes:

$$
E_{i+1}=\cos g_{i} E_{i}+\frac{j}{u_{i}} \sin g_{i} H_{i} \text {. }
$$

In a similar way, the following value for the magnetic field in the interface $i+1, i$ is obtained:

$$
H_{i+1}=j u_{i} \sin g_{i} E_{i}+\cos g_{i} H_{i} \text {. }
$$

The equations (5) and (6) can be written in a matrix form:

$$
\left[\begin{array}{l}
E_{i+1} \\
H_{i+1}
\end{array}\right]=\left[\begin{array}{cc}
\cos g_{i} & j \sin g_{i} / u_{i} \\
j u_{i} \sin g_{i} & \cos g_{i}
\end{array}\right]\left[\begin{array}{c}
E_{i} \\
H_{i}
\end{array}\right],
$$

or,

$$
\left[\begin{array}{l}
E_{i+1} \\
H_{i+1}
\end{array}\right]=M_{i}\left[\begin{array}{l}
E_{i} \\
H_{i}
\end{array}\right],
$$

where the matrix $M_{i}$ is given by:

$$
M_{i}=\left[\begin{array}{cc}
\cos g_{i} & j \sin g_{i} / u_{i} \\
j u_{i} \sin g_{i} & \cos g_{i}
\end{array}\right] .
$$

The matrix $M_{i}$ of $2 \times 2$ elements contains all the details of the film $i$ and relates the values of $E$ and $H$ of one side of the film with the ones of the other side. It can also be written:

$$
\left[\begin{array}{l}
E_{i+2} \\
H_{i+2}
\end{array}\right]=M_{i+1}\left[\begin{array}{l}
E_{i+1} \\
H_{i+1}
\end{array}\right]=M_{i+1} M_{i}\left[\begin{array}{c}
E_{i} \\
H_{i}
\end{array}\right] .
$$

In a general way the values of the electric and magnetic field in the exit medium, can be related with the ones in the incidence medium through the equation:

$$
\left[\begin{array}{l}
E_{q} \\
H_{q}
\end{array}\right]=M_{q-1} M_{q-2 i} \cdots M_{2} M_{1}\left[\begin{array}{l}
E_{o} \\
H_{o}
\end{array}\right] .
$$

In this case, it was considered that $g_{o}=0$, reducing $M_{o}$ to the identity matrix.

\section{H. Transmission and absorption coefficients}

Now, it is necessary to calculate the values of the electric and magnetic fields of the incident, reflected and transmitted waves. To do that, it is necessary to go back to the equation (4) that, written in the matrix form, becomes:

$$
\left[\begin{array}{c}
E_{q}^{+} \\
E_{q}^{-}
\end{array}\right]=\frac{1}{2}\left[\begin{array}{cc}
1 & 1 / u_{q} \\
1 & -1 / u_{q}
\end{array}\right]\left[\begin{array}{c}
E_{q} \\
H_{q}
\end{array}\right],
$$

and

$$
\left[\begin{array}{l}
E_{o} \\
H_{o}
\end{array}\right]=\left[\begin{array}{c}
1 \\
u_{o}
\end{array}\right] E_{o}^{+},
$$

as $E_{o}^{-}=0$.

Combining the equations (8), (9) and (10), comes:

$$
\left[\begin{array}{c}
E_{q}^{+} \\
E_{q}^{-}
\end{array}\right]=\frac{1}{2}\left[\begin{array}{cc}
1 & 1 / u_{q} \\
1 & -1 / u_{q}
\end{array}\right] M_{q-1} \cdots M_{1}\left[\begin{array}{c}
1 \\
u_{o}
\end{array}\right] E_{o}^{+} .
$$

From equation (11), the transmission and reflection coefficients are:

$$
r=E_{q}^{-} / E_{q}^{+} \quad t=E_{o}^{+} / E_{q}^{+},
$$

and for the reflectivity and transmissivity comes respectively:

$$
R=|r|^{2}=\left|E_{q}^{-} / E_{q}^{+}\right|^{2} \quad T=\frac{u_{o}}{u_{q}} t=\frac{u_{o}}{u_{q}}\left|E_{o}^{+} / E_{q}^{+}\right|^{2} .
$$




\section{Fabry-Perot optical filter}

The Fabry-Perot optical filter is an optical interface constituted by two films of high refractive index placed at both sides of a film of low refractive index, as it is shown in Fig. 3.

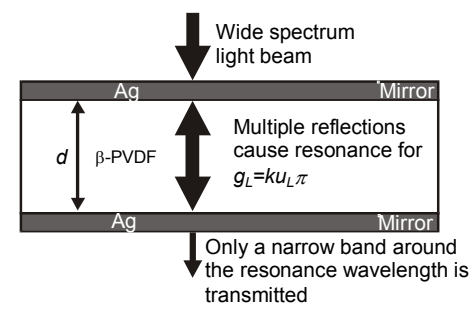

Fig. 3. Fabry-Perot optical filter.

In this case, the incidence and the exit media are constituted by air, whose refractive index is approximately equal to 1 . As $u_{q}=u_{o}=1$, Equation (11) becomes:

$$
\left[\begin{array}{c}
E_{q}^{+} \\
E_{q}^{-}
\end{array}\right]=\frac{1}{2}\left[\begin{array}{cc}
1 & 1 \\
1 & -1
\end{array}\right] M_{H} M_{L} M_{H} E_{o}^{+},
$$

where $M_{H}$ and $M_{L}$ represent the film matrixes of the high and low refractive index films respectively, and are given by equation (7). The transmissivity of the filter is then calculated using equation (12).

By analyzing equations (7) and (13), it is possible to conclude that the transmissivity of the Fabry-Perot optical filter depends, among other parameters, on the thickness of the low refractive index film. Actually, the resonant condition is achieved when the phase change in the low refractive index film is null, i. e. $\cos g_{L}=1$. This condition is achieved making $g_{L}=k \pi u_{L}, k=0,1,2, \ldots$ Therefore, in a Fabry-Perot optical filter the thickness of the resonance cavity determines the tuned wavelength.

\section{J. Piezoelectric Polymer}

As the best all-round piezoelectric polymer is still Poly(Vinylidene Fluoride) - PVDF, in its $\beta$-phase, this material was used as the resonance cavity of the Fabry-Perot optical filter.

PVDF is a polymer with interesting piezoelectric properties, which allows important electro-optical, electromechanical and biomedical applications. This polymer shows at least four crystalline phases called $\alpha, \beta, \gamma$ e $\delta$ [12-14]. The one with the best piezo- and pyroelectric properties, after poling, is the $\beta$ phase [14]. Until recently, this phase was exclusively obtained by mechanical stretching of films originally in the non-polar $\alpha$ phase $[12,13,15]$, which is the most stable one from a thermodynamic point of view and directly obtained from the melt. This process results in films mostly in the $\beta$ phase, but with a small percentage of $\alpha$ phase material [16-19]. Unoriented films exclusively in the $\beta$ phase were obtained from the crystallization of PVDF from solution with $\mathrm{N}, \mathrm{N}-$ Dimethyl Formamide or Dimethyl Acetamide at temperatures below $70^{\circ} \mathrm{C}$. The electromechanical properties of the film were improved by high temperature pressing. This process is being patented [20].
As the material is piezoelectric, two effects can be used in order to be implemented in the filter: the application of an acvoltage that will change the dimensions of the film or the application of a dc- voltage that will mainly act on the dipolar orientation and consequently in the polymer dielectric response. Whereas in the first case, the thickness variation will affect the wavelength response, in the second case, the transmittance will be affected.

In this investigation we will report mainly on the first approach. One of the main advantages of the present approach in comparison to the more traditional ones is that the variations in thickness and/or dielectric properties are due to variations at a molecular level, so, the changes are rather homogeneous within the samples. This reflects itself, for example, in the ability to maintain parallel mirrors, other wise a problem in conventional Fabry-Perot systems.

\section{DESIGN AND FABRICATION}

In order to fabricate the optical filter, two thin layers of silver used as the mirrors were deposited on both sides of the film (Fig. 3).

\section{A. Film deposition}

Fig. 4 shows a schematic diagram of the evaporation system used in the deposition of the silver films on the $\beta$-PVDF film.

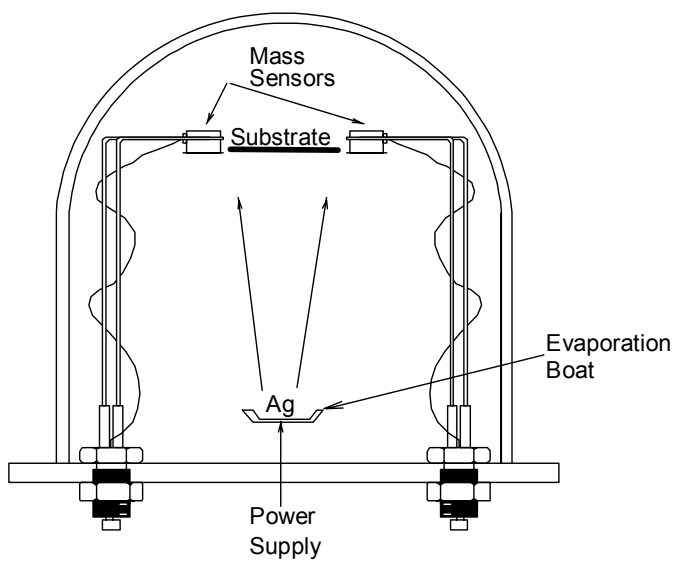

Fig. 4. Vacuum evaporator.

It consists in a vacuum chamber where the pressure reaches $10^{-6}$ mbar. An evaporation boat containing the silver sample is placed in its interior. The boat is heated by means of an electric current ranging between $100 \mathrm{~A}$ and $200 \mathrm{~A}$. At the top of the chamber the $\beta$-PVDF film, where the deposition is made, and two mass sensors are placed. In order to deposit the thin film of silver on the $\beta$-PVDF, the electrical power applied to the boat must be increased slowly. When the temperature of evaporation of the silver is reached, the mass sensors will indicate a change in the thickness (mass) attached to them. At this point, the electrical power must be kept constant. When the sensors indicate that the silver thickness is equal to $30 \mathrm{~nm}$, the power supply is switched off and the deposition of the first silver film is complete. The second silver film is deposited by 
turning upside down the $\beta$-PVDF film and repeating the previous procedure. Fig. 5 shows the Fabry-Perot optical filter.

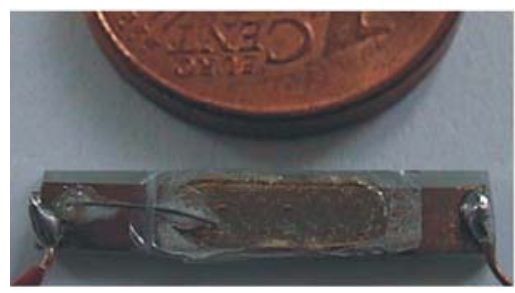

Fig. 5. The $1^{\text {st }}$ prototype tunable Fabry-Perot optical filter.

\section{B. Readout electronics}

In order to read the filter response, a monochromatic light source with adjustable wavelength and a photodiode was used. The main problem of a photodiode readout circuit is its performance relatively to the diversity of noisy signals that are into play. Ideally, a photodetector responds to a photon flow $\Phi$ with an optic power $P=h v \Phi$, generating an electric current proportional to the flow:

$$
i_{p}=\eta e \Phi=R P,
$$

where $h$ is the constant of Planck, $v$ is the frequency of the incident light, $e$ is the electron charge, $\eta$ is the quantum efficiency of the device and $R=(\eta e) /(h v)$ is its responsivity. In reality, the device generates a random electric current $i$ whose value changes around its average value $\bar{i}=i_{p}$. These random fluctuations, that can be seen as noise, are characterized by its standard deviation $\sigma_{i}$ where $\sigma_{i}^{2}=\left\langle(i-\bar{i})^{2}\right\rangle$. For a zero average current, the standard deviation is equal to the rms value of the current, i. e., $\sigma_{i}=\sqrt{\left\langle i^{2}\right\rangle}$. Some noise sources are inherent to the photon detection process [21]:

- Photon noise. The most basic source of noise is associated with the fact that the photons that arrive to the detector are random. This process is normally described by the statistical distribution of Poisson [22].

- Photoelectron noise. For a detector with quantum efficiency $\eta<1$, each photon generates an electron-hole pair with the probability $\eta$, but it fails the objective with the probability $1-\eta$. Due to its randomness, this process of carrier generation acts as a noise source.

- Photocurrent noise. The flow of photoelectrons causes an electric current in the circuit, where the photodetector is placed. It depends, beyond the properties of the photoelectron flow, on the time response of the photodetector.

- Readout circuit noise. The components that form the readout circuit, such as resistors or transistors, contribute to the noise of the reception circuit.

- Background noise. The background noise is caused by the photons that reach the detector proceeding from undesirable external light sources.
- Dark current. The dark current is present even in the absence of light. This noise results from the fact that electron-hole pairs are randomly generated by thermal processes or tunneling effect.

- $\quad 1 / f$ noise. This form of noise is perhaps the most studied and at the same time the worse understood. It is characterized by its power spectrum, which decreases quickly with the frequency $(1 / f)$.

Concluding, the signal that reaches the detector has the intrinsic photon noise. The photoelectric effect converts the photons into electron-hole pairs. In this conversion, the average value of the signal decreases $\eta$ times. The noise also decreases, but less than the signal. This makes that the signal to noise ratio of the photoelectrons is lower than the one of the incident photons. At this level, the noise caused by the dark current, the background noise and the $1 / f$ noise are added. Finally, the noise of the readout circuit is added at the point in which the current is read. From the above discussion, it is possible to identify three noise sources that, having some care in the design of the experimental setup, it is possible to reduce substantially: the readout circuit noise, the background noise and the dark current. In order to reduce the readout circuit noise, it must be used precision resistors, with low temperature coefficients and low offset operational amplifiers. In order to reduce the background noise, special care must be taken with the external light sources. In the present case, the experiments were made in a dark room and the optical setup was placed inside a black box. The dark current is exponentially related with the inverse bias voltage applied to the photodetector [21]. In order to reduce this noise, the photodetector must be biased with a voltage as low as possible. Fig. 6 shows a circuit that biases the photodiode with a null inverse voltage. Its implementation is shown in Fig. 7.

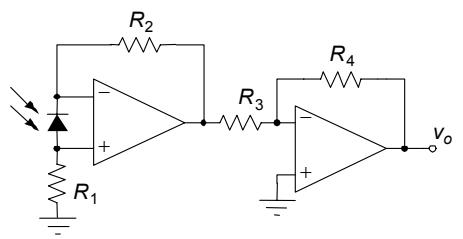

Fig. 6. Readout electronic circuit.

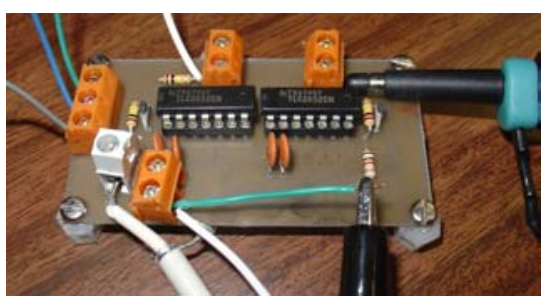

Fig. 7. A photograph of the readout electronic circuit.

The photocurrent $i$ generated in the photodiode passes through $R_{1}$ and $R_{2}$, making that the voltage at the output of the first operational amplifier is $v_{o 1}=-\left(R_{1}+R_{2}\right) i$. The voltage at the circuit output will then be $v_{o}=\left(R_{1}+R_{2}\right)\left(R_{4} / R_{3}\right) i$. 


\section{EXPERIMENTAL RESULTS}

The experimental arrangement used in the measurements comprises a $250 \mathrm{~W}$ quartz tungsten halogen lamp with a monochromator ORIEL Cornerstone $130^{\mathrm{TM}} \quad(1200 \mathrm{~g} / \mathrm{mm}$ grating with a spectral dispersion of $6.6 \mathrm{~nm} / \mathrm{mm}$ and a spectral resolution of $0.5 \mathrm{~nm}$ ) that is used as light source. An optical fiber is also used to direct the light into the black box. The photodiode is the low dark current S1787 from Hamamatsu.

In the first experiment, a $5 \mathrm{~V}$ sine wave was applied to the mirrors of the Fabry-Perot optical filter. Fig. 8 shows the measured values for several incident light wavelengths. The conclusion that can be taken from this figure is that for each wavelength, the optical response of the filter changes with the applied voltage.

Fig. 9 shows the response of the filter as a function of the wavelength. In order to obtain this graph, a similar procedure of the Fig. 8 was used for each wavelength. When the voltage applied to the filter crossed some values, its response was taken, originating the graph of Fig. 9. From this figure, it is possible to see once again that the filter response changes systematically with the applied voltage.

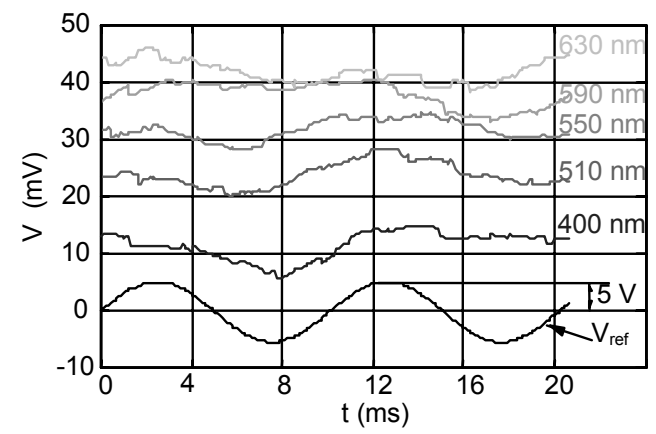

Fig. 8: System response for several wavelengths, when the voltage $V_{\text {ref }}$ is applies to the Fabry-Perot filter.

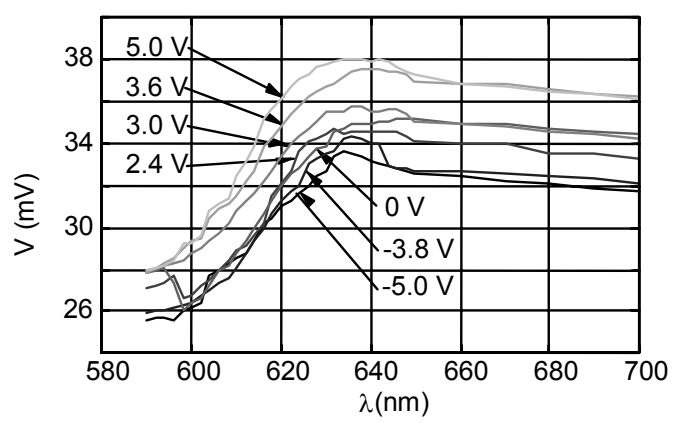

Fig. 9: System response in function of the wavelength, when the sinusoidal voltage applied to the filter has some specific values (shown in figure).

\section{CONCLUSIONS}

The concept, a theoretical analysis, the fabrication and the performance of a tunable Fabry-Perot optical filter with a piezoelectric polymer as the resonance cavity were proved and reported. The filter can be an integrated part of portable (bio)chemical micro total analysis systems once it enables the spectrophotometric measurements using only white light source. The major advantage of this design is the ability to maintain parallel mirrors, during the tuning of the filter, due to the material in its cavity, which features thickness variations at a molecular level. As a future work, it will be fabricated a tunable Fabry-Perot filter based on $\beta$-PVDF, with a thickness near $300 \mathrm{~nm}$. The theoretical analysis shows that with this piezoelectric material it is possible to obtain a tunable filter that acts in the range of the biomolecules reported in the introduction of this article.

\section{ACKNOWLEDGMENT}

Support for this research was provided by the Engineering School of University of Minho (program IN2TEC), the R\&D Centre Algoritmi of University of Minho and the Foundation for Science and Technology (Grant POCI/CTM/59425/2004).

\section{REFERENCES}

[1] P. Connolly, "Clinical diagnostics opportunities for biosensors and bioelectronics," Biosensors \& Bioelectronics, vol. 10, pp. 1-6, 1995.

[2] S. K. Strasinger and M. S. Di Lorenzo, Urianalysis and Body Fluids, 4th ed., F. A. Davis Company, Philadelphia, PA. 2001.

[3] http://www.hypoguard.com/diascreen_reagent_strips.html.

[4] J. C. Todd, A. H. Sanford and I. Davidson, Clinical diagnosis and management, 17th ed., W. B. Saunders Company, 1984.

[5] Biochemical and organic reagents, Sigma, 2005.

[6] M. Born and E. Wolf, Principles of optics; electromagnetic theory of propagation, interference and direction of light, Pergamon, Oxford, 9th edition, 1999.

[7] H. A. Macleod, Thin-Film Optical Filters, Institute of Physics Publishing, Bristol and Philadelphia, 3rd edition, 2001.

[8] P. Yeh, Optical Waves in Layered Media, Wiley, 1988.

[9] I. J. Hodgkinson and Q. H. Wu, Birefringent Thin Films and Polarizing Elements, World Scientific, Singapore, 1997.

[10] D. W. de Lima Monteiro, G. Vdovin, J. G. Rocha, V. P. Iordanov, M. Y. Loktev and P. M. Sarro, "Customized CMOS wavefront sensor," In Proc. SPIE, vol. 4493, pp. 88-99, 2002.

[11] D. P. Poenar, Thin Film Colour Sensors, Delft University Press, The Netherlands, 1996.

[12] A.J. Lovinger, in Developments in Crystalline Polymers, edited by I.D.C. Basset, Elsevier Applied Science, London, 1982.

[13] A.J. Lovinger, Science, vol. 220 pp. 1115, 1983.

[14] G.M Sessler, Topics in Applied Physics: Electrets, 2nd Ed., Springer, Berlin Heidelberg New York, 1987.

[15] K. Matsushige, K. Nagata, S. Imada, and K. Takemura, Polymer, vol. 21 pp. 1391, 1980.

[16] V. Sencadas, S. Lanceros-Mendez and J.F. Mano, Thermochimica Acta, vol. 424, pp. 201, 2004.

[17] S. Lanceros-Mendez, J.F. Mano, A.M. Costa and V.H. Schmidt, J. Macrom. Sci.- Phys., vol. B40, pp.517, 2001.

[18] S. Lanceros-Mendez, M.V. Moreira, J.F. Mano, V.H. Schmidt and G. Bohannan, Ferroelectrics, vol. 273, pp. 15, 2002.

[19] R. Gregorio Jr and M. Cestari, Journal of Polymer Science: Part B: Polymer Physics, vol. 32, pp.859, 1994.

[20] S. Lanceros-Mendez, V. Sencadas and R. Gregorio Filho, Portuguese patent $n .^{\circ} 103318$.

[21] J. G. Rocha and S. Lanceros-Mendez, Scintillator based $x$-ray detectors, Encyclopedia of Sensors, American Scientific Publishers, 2006 - To be published.

[22] B. E. A. Saleh and M. C. Teich, Fundamentals of Photonics, John Wiley and Sons, 1991. 